\title{
Development of conductor-backed coplanar waveguide moisture sensor for oil palm fruit
}

\begin{abstract}
The analysis, design and performance of a conductor-backed coplanar waveguide (CBCPW) moisture sensor are described. The sensor can be used to determine the moisture content (MC in per cent) in oil palm fruit and other agricultural products. The sensor consists of three parts - the coupling system representing the transition between the coaxial line and the CBCPW, the two-layer structures of the CBCPW and the sensing area with its semi-infinite three-layer CBCPW structure. A relationship between the scattering parameter S 21 or attenuation of the sensor and the moisture content of the sample has been developed. The reflection and transmission phenomena in the sensor structure can be represented by a signal flow graph and can be simplified by the use of non-touching loop rules. The calculation of S 21 is based on the quasi-transverse electromagnetic mode approximation. This work also studies the effect of the thickness of the protective layer and the size of the gap between the conducting line and the upper ground plane on the sensitivity and dynamic range of the sensor. Close agreement between the theoretical prediction and experimental results is obtained. The sensitivity and accuracy of the sensors are about $0.5 \mathrm{~dB} / \mathrm{MC}$ and $1 \%$ (unit MC, wet basis), respectively. The sensor is suitable for development as a complete instrument and can be used for quality assessments of oil palm fruit and other agricultural products.
\end{abstract}

Keyword: Conductor-backed coplanar waveguide; Moisture sensors; Oil palm fruit 